\title{
Comparison of the effectiveness of ultrasound elastography with that of conventional ultrasound for differential diagnosis of thyroid lesions with suspicious ultrasound features
}

\author{
JINRU YANG, YAN SONG, WEI WEI, LITAO RUAN and HONG AI \\ Department of Ultrasonics, The First Affiliated Hospital of Medical College, \\ Xi'an Jiaotong University, Xi'an, Shanxi 710061, P.R. China \\ Received September 14, 2015; Accepted February 23, 2017
}

DOI: $10.3892 / \mathrm{ol} .2017 .6644$

\begin{abstract}
Ultrasound elastography (USE) has been used for quantitative imaging since the early 1990s. The aim of the present study was to compare the effectiveness of USE with that of conventional ultrasound in the differential diagnosis of benign vs. malignant thyroid nodules. USE was performed on 150 thyroid nodules in 123 patients. USE demonstrated a lower grade of elasticity (grade I/II) in $86 \%$ of benign nodules and a decreased strain ratio $(2.30 \pm 1.01)$ compared with malignant nodules. USE of malignant nodules exhibited a higher grade of elasticity (grade III/IV) in $90 \%$ of nodules and an increased strain ratio $(6.39 \pm 2.50)$. The results of the present study indicate that USE and strain ratio may be useful in the differential diagnosis of thyroid nodules.
\end{abstract}

\section{Introduction}

The worldwide incidence of thyroid malignancy has increased $>2$-fold since the 1970s (1). Improvements in the diagnostic capabilities for microcarcinomas, for which long-term outcomes are generally excellent, has contributed to this increase $(2,3)$. Surgical excision is the gold-standard method for differentiating malignant from benign thyroid disease. Ultrasound (US) -guided fine needle aspiration (FNA) with cytology (FNAC) is not warranted in the evaluation of all thyroid nodules, $>95 \%$ of which are benign (4-6).

US is non-invasive and does not expose patients to ionizing radiation, and high-resolution US has become the gold-standard modality for imaging thyroid nodules. In its conventional two-dimensional application, US is useful for guiding FNA,

Correspondence to: Dr Jinru Yang, Department of Ultrasonics, The First Affiliated Hospital of Medical College, Xi'an Jiaotong University, 277 Yanta West Road, Xi'an, Shanxi 710061, P.R. China E-mail: yangjinru13@yeah.net

Key words: ultrasonography, elastography, thyroid nodules, papillary carcinoma, nodular thyroid, ultrasound elastography for distinguishing cystic from solid nodules and for elucidating other aspects of nodular morphology. Malignancy is more likely in nodules exhibiting hypoechogenicity, punctate microcalcifications, elevated central color flow, rough edges, a high anteroposterior to transverse diameter or lack of a halo (7). However, as a number of these features lack accuracy in distinguishing benign lesions from malignancy, the usefulness of conventional US is limited (8-11).

Tissue elasticity is another important index for assessing the risk of malignancy. When a thyroid nodule is palpable, an elevated index of suspicion is justified when physical examination reveals that it is firm compared with the surrounding tissue. Only a minority of thyroid nodules are palpable, however, and assessment of firmness is subjective and influenced markedly by the location and size of the lesion (4). Although the Doppler mode adds a valuable dimension to conventional US, it provides no useful information with respect to nodule elasticity (4,12-14).

First utilized to evaluate thyroid nodules by Lyshchik et al (15), US elastography (USE) has been used in other tissues since the early 1990s (16,17). By quantifying the degree of distortion resulting from a force applied to tissue, USE generates estimates of the stiffness/elasticity of a tissue and comparisons of the stiffness of different tissues $(4,7)$. Consequently, the technique has been termed 'electronic palpation' (4). In comparison with soft nodules, firm nodules are less prone to deformation when compressive forces are applied (i.e. they are less elastic) and the lower elasticity is associated with the risk of malignancy $(4,7,18-20)$. In addition to selecting high-risk nodules for further evaluation by FNAC, the elasticity information from USE may aid in the diagnosis of nodules that are deemed cytologically indeterminate (21).

The aim of the present study was therefore to compare effectiveness of USE with that of conventional US in the differential diagnosis of benign vs. malignant thyroid nodules.

\section{Materials and methods}

Patients. The present retrospective study was approved by the Institutional Review Board of The First Affiliated Hospital of Medical College (Xi'an Jiaotong University, Xi'an, China) who waived the requirement for informed consent due to the 
retrospective nature of the study. A total of 123 consecutive patients [101 females and 22 males; age range, between 23 and 56 years (median, 40 years)] who underwent examination at The First Affiliated Hospital of Medical College for thyroid nodules from January 2012 to February 2013 were included. None of these patients had any environmental exposure or genetic predisposition to thyroid carcinoma.

The nodule diameters ranged between 0.5 and $1.6 \mathrm{~cm}$. All patients underwent routine preoperative conventional US and elastography examinations. All nodules were confirmed by pathology. The length of time between US and surgery was $\sim 2$ weeks.

A number of the 150 nodules were surgically excised and a number of diagnoses were made using FNAC. Accepted criteria for FNAC biopsy results were used and the pathologists who made the histological diagnosis were blinded to the results of conventional US and USE. No prior imaging was reviewed before enrollment.

Inclusion/exclusion criteria. To be included in the present study, each nodule from each patient was required to meet the following requirements: A maximum diameter $<2 \mathrm{~cm}$, and no obvious abnormal echo within the surrounding thyroid tissue.

If the nodule volume was too large (maximum length $>2 \mathrm{~cm}$ ), the cystic component of a nodule was $>50 \%$ of the total volume, or the nodule was located within the thyroidal isthmus, the patient was excluded, because the above conditions would result in poor elastographic image quality.

On the basis of parameters used in the routine US diagnosis (22), all masses were suspicious for malignancy if they contained solid hypoechoes, unclear boundary, no peripheral halo, irregular shape, posterior echo attenuation, an aspect ratio $\geq 1$, increased perfusion or diffuse internal sand-like microcalcifications. Therefore, those patients with such nodules were considered high-risk patients. Nodules suspected of malignancy were included in the present study. Nodules that were highly suspicious for malignant tumors were surgically resected. The subjects were also included in the study group due to results of indeterminate FNAC and referral for surgery. Of 180 nodules evaluated, 30 did not meet the inclusion criteria and were therefore excluded. The remaining 150 nodules from 123 patients were included in the present study for further analysis (Fig. 1).

The presence of a single US criterion for malignancy was sufficient for inclusion. Not all high-risk nodules exhibit all the features of malignancy, i.e. a number exhibited only one or two malignant features. Not all nodules suspicious for malignancy exhibited internal microcalcifications. Masses with solid hypoechoes, unclear boundary, irregular shape, posterior echo attenuation, length/width ratio $\geq 1$ and diffuse microcalcifications were diagnosed as malignant lesions. Non-high-risk subjects did not undergo USE. Only high-risk subjects were assessed using USE.

In order to avoid subjectivity of scoring, each patient was diagnosed by two radiologists with $>5$ years of experience in US diagnosis. If the two radiologists differed in their diagnoses, they conferred to reach a final consensus. The radiologists were blinded to the clinical data, but knew the results of the traditional US.

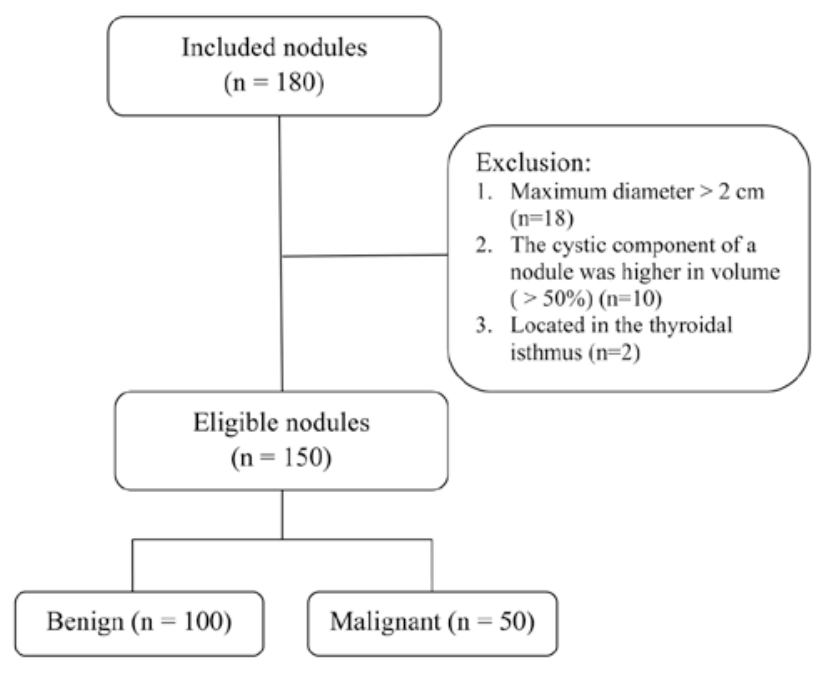

Figure 1. Flow chart of study selection.

Instrumentation. Elastographic data were obtained using an EUB-7500 platform and Hi Vision Preirus color Doppler ultrasonography instrument (both Hitachi, Ltd., Tokyo, Japan) equipped with real-time USE (RE) software. The probe frequency was between 6 and $13 \mathrm{MHz}$.

US evaluation. The lesions were first examined by grayscale US, and routine horizontal and vertical scans were performed. Tumor location, size, shape, boundary, internal echoes and cervical lymph nodes were evaluated. Subsequently, color and pulsed Doppler ultrasonography were performed to observe blood flow within the lesions, followed by elastography. The sampling frame [i.e. the region of interest (ROI)] was increased between 2- and 3-fold compared with the lesion. A handheld probe was placed at the site of the lesion and the pressure was maintained at between 3 and 4 according to the digital display. The dual-amplitude real-time display function was used to display the two-dimensional and elastography maps simultaneously to enable comparison between the hardness of each lesion and its surrounding normal thyroid tissues. When stable elasticity images were obtained, ROIs were selected in both the lesional area and surrounding normal thyroid tissues to calculate the strain ratio between the two.

Hardness grade of lesions on elastography images. According to the color display of the lesions (i.e. relative hardness), the lesion elasticity was divided into four grades (23): Grade I, lesions and surrounding tissues had uniform green color; grade II, lesions were mainly green, and the periphery and a small amount of inside areas were blue; grade III, lesions were mainly blue in color, exhibiting disordered interlacing blue and green color; and grade IV, lesions were completely blue.

Statistical analysis. Data are represented as the median with an inter-quartile range (IQR, the range between the 25th and 75 th percentiles) for continuous variables due to non-normal data distributions, and as frequencies with percentages for categorical variables. The comparisons between benign and 
Table I. Demographic and pathological characteristics of patients and thyroid nodules.

\begin{tabular}{lcc}
\hline Characteristic & Benign & Malignant \\
\hline Patient characteristics & & $50^{\mathrm{a}}$ \\
No. of patients & 73 & $36(31-40)$ \\
Median age, years (IQR) & $44(39-48)$ & $37(74.0)$ \\
Sex, n (\%) & & $13(26.0)$ \\
Female & $64(87.7)$ & \\
Male & $9(12.3)$ & 50 \\
Thyroid nodule characteristics & & $8.0(6.0-9.0)$ \\
No. of thyroid nodules & 100 & \\
Median size, mm (IQR) & $9.0(8.0-11.0)$ & $48(96.0)$ \\
Pathology, n (\%) & $0(0.0)$ & $1(2.0)$ \\
Papillary carcinoma & $0(0.0)$ & $1(2.0)$ \\
Follicular carcinoma & $0(0.0)$ & $0(0.0)$ \\
Medullary carcinoma & $70(70.0)$ & $0(0.0)$ \\
Nodular goiter & $28(28.0)$ & $0(0.0)$ \\
Adenoma & $2(2.0)$ & $<0.001^{\mathrm{b}}$ \\
Hürthle cell thyroid tumor & & \\
\hline
\end{tabular}

${ }^{\mathrm{a}} \mathrm{A}$ total of 6 patients in the malignant group exhibited malignant and benign thyroid nodules. ${ }^{\mathrm{b}} \mathrm{P}$-value determined by Wilcoxon's rank sum test. ${ }^{\mathrm{c}} \mathrm{P}$-value determined by $\chi^{2}$ test. ${ }^{\mathrm{d}} \mathrm{P}$-value determined by Fisher's exact test. IQR, interquartile range.

Table II. Distribution of elastography grades between benign and malignant thyroid nodules.

\begin{tabular}{lcc}
\hline Elastography grade & Benign, n (\%) & Malignant, n (\%) \\
\hline I & $46 / 100(46.0)$ & $1 / 50(2.0)$ \\
II & $40 / 100(40.0)$ & $4 / 50(8.0)$ \\
III & $10 / 100(10.0)$ & $15 / 50(30.0)$ \\
IV & $4 / 100(4.0)$ & $30 / 50(60.0)$ \\
\hline
\end{tabular}

A significant association between higher elastography grade and malignant nodules was identified $\left(\mathrm{P}<0.001\right.$ determined by $\chi^{2}$ test).

malignant groups were performed by Wilcoxon's rank sum test for continuous data and by $\chi^{2}$ test or Fisher's exact test for categorical data. The receiver operating characteristic (ROC) curves were used to examine the diagnostic performance characteristics of USE (i.e. grade and strain ratio) vs. traditional US to distinguish benign and malignant thyroid nodules. To compare the distinguishing ability among three parameters, the point estimate with $95 \%$ confidence interval (CI) of the area under the ROC curve (AUC) was provided as an index to compare global test performance; pairwise comparison of AUC among three parameters was also performed. Youden's index was used to determine the optimal cut-off point to differentiate benign from malignant thyroid nodules, which were determined by maximizing the sum of sensitivity and specificity (24). The corresponding sensitivity, specificity, positive predictive value (PPV), negative predictive value (NPV) and accuracy were provided at the optimal threshold values. The statistical analyses were performed using SAS software (version 9.2; SAS Institute Inc., Cary, NC, USA), and two-tailed $\mathrm{P}<0.05$ was considered to indicate a statistically significant difference.

\section{Results}

Demographic and pathological characteristics of patients and thyroid nodules. The characteristics of patients and thyroid nodules are summarized in Table I. Of 123 patients (a total of 150 thyroid nodules), 50 patients were diagnosed with malignant nodules (papillary carcinoma, $n=48$; follicular carcinoma, $n=1$; medullary carcinoma, $n=1$ ) and 100 patients were diagnosed with benign nodules (nodular goiter, $n=70$; adenoma, $n=28$; Hürthle cell thyroid tumors, $n=2$ ). A total of 50 patients were identified to exhibit $\geq 1$ malignant thyroid nodule ( 6 patients exhibited malignant and benign thyroid nodules) and 73 patients were identified to exhibit all benign thyroid nodules. The patients in the malignant group were significantly younger compared with those in the benign group [median (IQR), 36 (31-40) years vs. 44 (39-48) years; $\mathrm{P}<0.001]$. The malignant thyroid nodules were significantly smaller compared with the benign nodules [median (IQR), 8.0 (6.0-9.0) $\mathrm{mm}$ vs. 9.0 (8.0-11.0) $\mathrm{mm} ; \mathrm{P}<0.001]$.

Elastography grade and pathological results of thyroid nodules. The distribution of elastography grades (25) between benign and malignant thyroid nodules are presented in Table II. In total, $86(86 \%)$ benign thyroid nodules were classified as grade I and II, and 45 (90\%) malignant thyroid nodules were classified as grade III and IV. A significant association between higher elastography grade and malignant thyroid nodules was identified $(\mathrm{P}<0.001)$. Representative USE images are presented in Figs. 2 and 3. 
Table III. Comparison of diagnostic performance in distinguishing benign from malignant thyroid nodules using elastography grade, strain ratio and traditional ultrasound.

\begin{tabular}{lccccc}
\hline Diagnostic method & Sensitivity, \% (CI) & Specificity, \% (CI) & PPV, \% (CI) & NPV, \% (CI) & Accuracy, \% (CI) \\
\hline Elastography grade $^{\mathrm{a}}$ & $90.0(81.7-98.3)$ & $86.0(79.2-92.8)$ & $76.3(65.4-87.1)$ & $94.5(89.8-99.2)$ & $87.3(82.0-92.7)$ \\
Strain ratio $^{\mathrm{b}}$ & $88.0(79.0-97.0)$ & $93.0(88.0-98.0)$ & $86.3(76.8-95.7)$ & $93.9(89.2-98.6)$ & $91.3(86.8-95.8)$ \\
Traditional ultrasound $^{\mathrm{c}}$ & $82.0(71.4-92.7)$ & $69.0(59.9-78.1)$ & $56.9(45.5-68.4)$ & $88.5(81.4-95.6)$ & $73.3(66.3-80.4)$ \\
\hline
\end{tabular}

The criteria for detecting malignant thyroid nodules were as follows: ${ }^{a}$ Elastography grade of $\geq \mathrm{III}$; ${ }^{\mathrm{b}}$ strain ratio $\geq 3.68$; and ${ }^{\mathrm{c}}$ ultrasound imaging characteristics as follows: Irregular shape and unclear boundary, no peripheral halo, an aspect ratio $\geq 1$, hypoechogenicity, internal sand-like calcification. PPV, positive predictive value; NPV, negative predictive value; CI, asymptotic $95 \%$ confidence interval.

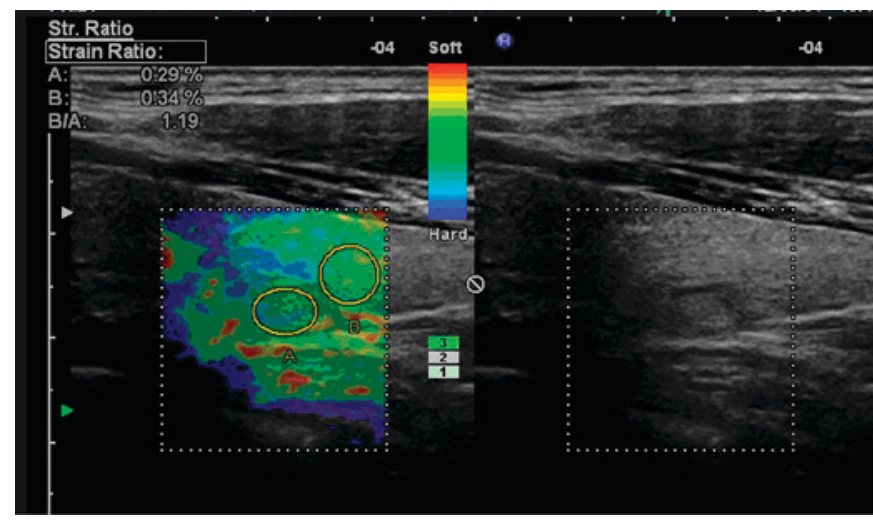

Figure 2. Grayscale sonographic map and elastographic map of benign thyroid nodules. Left; elastographic map indicating that the elastic strain ratio is 1.19. Right; the two-dimensional map illustrates a 7x6 $\mathrm{mm}$ nodule with medium echo, clear boundary and halo. Surgical pathology; adenomatous nodule goiter.

Strain ratio and pathological results of thyroid nodules. The distribution of strain ratio between benign and malignant thyroid nodules is presented in Fig. 4. The malignant thyroid nodules exhibited a significantly increased strain ratio compared with that of benign nodules [median (IQR), 6.36 (4.62-8.42) vs. 2.12 (1.61-2.71); $\mathrm{P}<0.001]$.

ROC curve analysis using elastography grade and strain ratio to distinguish benign and malignant thyroid nodules. As elastography grade and strain ratio demonstrated significant differences between benign and malignant thyroid nodules, further ROC curve analysis was performed to compare the discriminating ability between these two diagnostic approaches and traditional US (Fig. 5). Compared with traditional US, elastography grade and strain ratio demonstrated significantly increased AUCs $(\mathrm{P}<0.001$; Fig. 5). Additionally, the strain ratio exhibited an improved diagnostic utility compared with traditional US ( $\mathrm{P}<0.001$; Fig. 5). However, no significant difference was identified for AUC between elastography grade and strain ratio $(\mathrm{P}=0.902)$. Furthermore, the corresponding sensitivity, specificity, PPV, NPV and accuracy were $90.0,86.0,76.3,94.5$ and $87.3 \%$ for elastography grade, 88.0, 93.0, 86.3, 93.9 and $91.3 \%$ for strain ratio, and 82.0, $69.0,56.9,88.5$ and $73.3 \%$ for traditional US, respectively (Table III). The optimal threshold values corresponded to the highest sum of sensitivity and specificity.

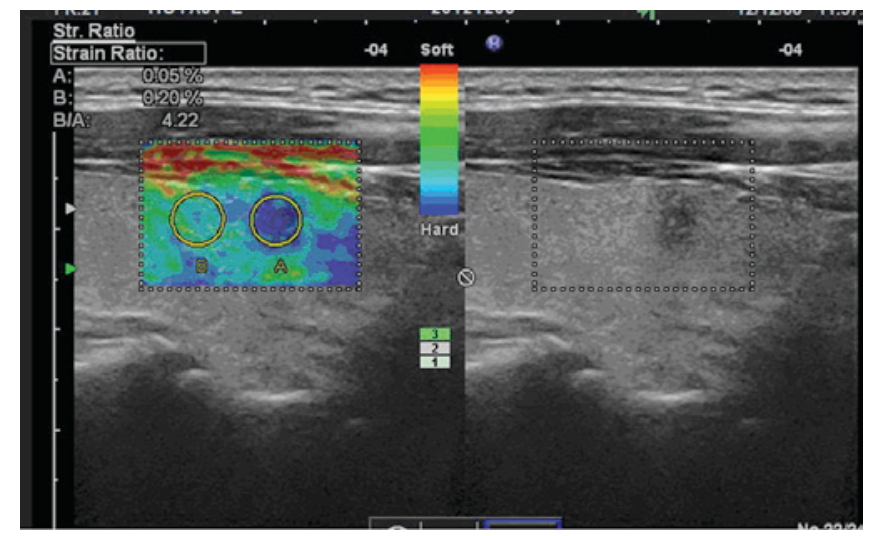

Figure 3. Grayscale sonographic map and elastographic map of malignant thyroid nodules. Left; elastographic map indicating that the elastic strain ratio is 4.22. Right; the two-dimensional map illustrates a $6 \times 9 \mathrm{~mm}$ hypoechoic nodule with irregular shape and no halo. Surgical pathology; papillary thyroid carcinoma.

\section{Discussion}

US serves an important function in the detection, diagnosis, prognosis and follow-up of a thyroid mass. However, its value for the differentiation of benign from malignant masses is limited. Microcalcifications, aspect ratio $\geq 1$ and ragged edge have been used as specific characteristics of malignant tumors $(23,26)$. In recent years, the incidence of micropapillary thyroid carcinoma has increased $(1,2)$. However, prognosis for this condition is usually favorable provided that an early diagnosis is made, usually via FNA, although it is an invasive technique (4-6). Completely non-invasive real-time USE is able to differentiate thyroid cancer from benignity by assessing tissue hardness and elasticity, by grading the elasticity of thyroid nodules, and by measuring the strain ratio (20,27-30).

In the present study, USE of 150 thyroid nodules demonstrated primarily benign nodular goiter $(n=100)$ with nodules exhibiting a soft texture and significant deformation under compression. Additionally, these nodules exhibited a large number of follicles filled with colloid. Nodular texture was soft, and USE demonstrated a lower grade of elasticity (grade I/II) in $86 \%$ of benign nodules and a low strain ratio $(2.30 \pm 1.01)$. Of the 100 nodules in the benign group, 86 nodules had an elasticity score $<3$. However, 10 benign nodules had a score of 3 , and 4 benign nodules had a score of 4 and were misdiagnosed as false positives. A number of nodules exhibited calcification, 


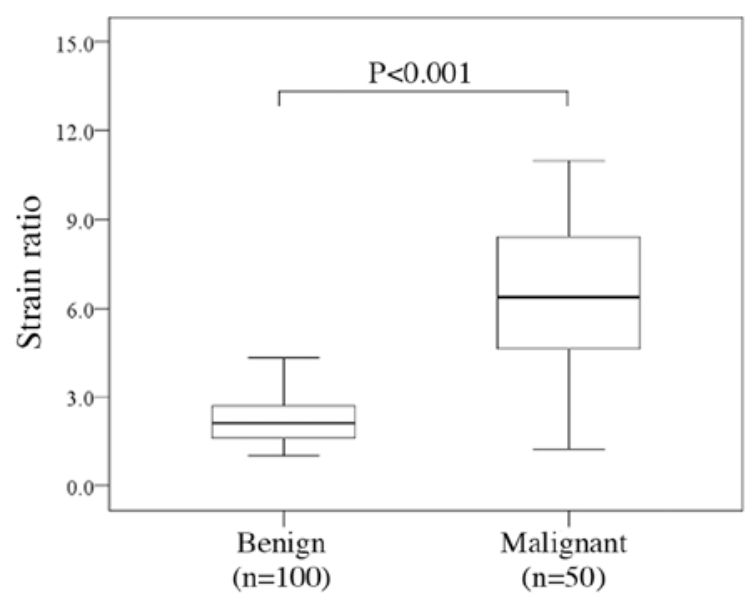

Figure 4. Distribution of strain ratios between benign and malignant thyroid nodules. The malignant thyroid nodules exhibit significantly increased strain ratio compared with the benign nodules [median (interquartile range), 6.36 (4.62-8.42) vs. $2.12(1.61-2.71) ; \mathrm{P}<0.001]$

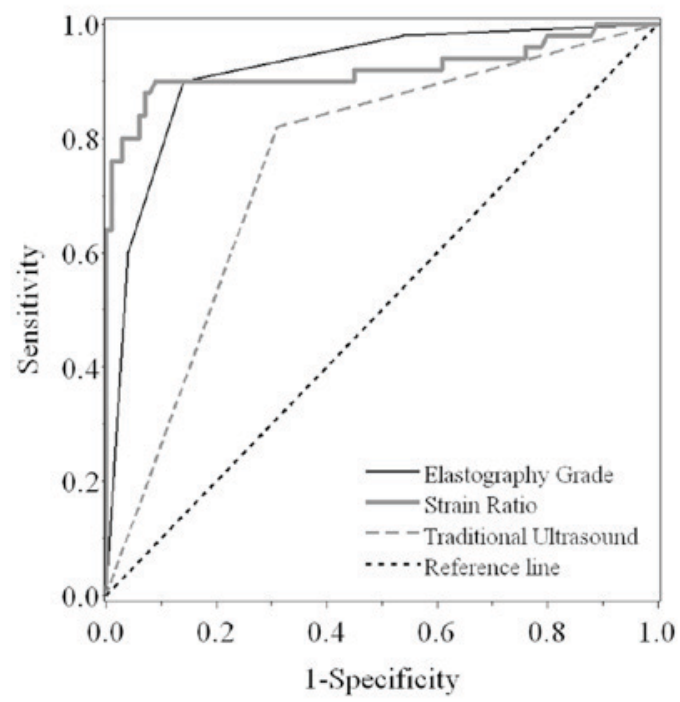

Figure 5. Receiver operating characteristic curve analyses. The specificity and sensitivity of using elastography grade, strain ratio and traditional ultrasound to distinguish benign from malignant thyroid nodules were compared The AUC was 0.918 (95\% CI, 0.872-0.965), 0.921 (95\% CI, 0.860-0.982) and 0.755 (95\% CI, 0.685-0.826) for elastography grade, strain ratio and traditional ultrasound, respectively. The AUCs of elastography grade and strain ratio were significantly increased compared with those of traditional ultrasound $(\mathrm{P}<0.001)$. However, no significant difference in AUC was identified between elastography grade and strain ratio $(\mathrm{P}=0.902)$. AUC, area under the curve; CI, confidence interval.

causing increased stiffness, and nodule position may also have affected the elasticity score.

In contrast, malignant nodules in the present study were harder with less deformation under compression. The pathological results demonstrated primarily papillary carcinoma, with an interstitium accompanied by sand bodies yielding a hard texture. USE of malignant nodules demonstrated a higher grade of elasticity (grade III/IV) in $90 \%$ of nodules and an increased strain ratio $(6.39 \pm 2.50)$. Of the 50 nodules in the malignant group, $45(90 \%)$ malignant thyroid nodules were classified as grade III and IV. The elasticity scores of 15 malignant nodules was 3 and 30 had elasticity scores of 4 . The elasticity scores of the remaining five malignant nodules were 1 in one nodule and 2 in four nodules; these were misdiagnosed as false negatives. False negative results of USE included a nodule whose histopathology exhibited medullary thyroid cancer and another whose histopathology exhibited follicular carcinoma. Cancer cells in a follicle-like arrangement with deposition of amyloid-like substance within the interstitium may create a soft texture causing a false negative USE reading. False negative results on USE may also have resulted from large quantities of fluid in cystic nodules, which may have decreased their stiffness.

USE is particularly appropriate for the thyroid, because of its accessible anatomic position $(7,15,21,31,32)$. A meta-analysis of eight studies evaluating real-time elastography (RTE) and consisting a total of 639 thyroid nodules suggests that RTE is able to differentiate malignancy accurately compared with FNAC (33). Specifically, the aforementioned meta-analysis associated malignancy with stiffness with a sensitivity and specificity of 92\% (CI 88-96\%) and 90\% (CI 85-95\%), respectively. Additional trials have yielded similar results for USE evaluation of thyroid nodules in groups of patients who were later identified to have a high incidence of malignancy (34).

A previous study by Vidal-Casariego et al (35) failed to demonstrate accurate differentiation of malignant and benign nodules when USE was used on nodular goiter patients at low risk of thyroid malignancy. However, this study involved a total of 128 patients and examination of larger numbers of patients across multiple risk groups is required prior to drawing definitive conclusions regarding the utility of USE across risk groups. Additionally, the accuracy of USE has been questioned in the setting of diffuse thyroid disease, such as chronic Hashimoto thyroiditis $(5,36)$. At the same time, efforts to standardize USE protocols and enhance USE imaging may lead to improved accuracy. These efforts include a proposed classification system using linear discriminant analysis to select possible malignant nodules detected by USE (37) and an innovation known as micropure imaging (38).

The present study has identified that measuring the strain ratio in USE may assist in the differentiation of benign from malignant nodules. ROC curves were plotted for 123 patients with benign and malignant nodules, and these provide a maximum Youden index of 0.81 , and a corresponding optimal cut-off point of the elastic strain ratio of 3.68. Consequently, 3.68 was selected as the threshold for differentiating benign from malignant thyroid nodules. This value differs from strain ratio threshold values in the literature possibly due to different measurement techniques and operating practices. When two threshold points are identified from two separate studies, it is necessary to determine whether study participants come from populations with different sociodemographics or clinical profiles, whether the diagnostic criteria of disease (i.e. malignant vs. benign) were different or whether the measurements of strain ratio (e.g. the instrument used and parameter settings) were different. Therefore, owing to different instruments and operating practices, the threshold values may vary. AUC was also used to evaluate the diagnostic accuracy; the AUC of the strain ratio value was 0.921, and the AUC of elasticity classification was 0.761 , demonstrating certain accuracy. 
The present study exhibited several limitations including its retrospective nature. In addition, the number of patients with follicular carcinoma and medullary carcinoma was low. Therefore, imaging features of follicular and medullary carcinoma require further study. Furthermore, the number of patients with papillary carcinoma was high. Therefore, future studies should utilize more diverse patient groups. On the basis of our inclusion and exclusion criteria, the present study was performed on pre-selected high-risk patients as only nodules suspected of being malignant were included. This pre-selection led to an increased rate of malignancy compared with the normal rate $(33 \%)$ in the present study population as the denominator for our $33 \%$ malignancy rate was a pre-selected population of thyroid nodules from high-risk patients. In contrast, the denominator for the $95 \%$ benign nodule rate is the general population (4-6). It is anticipated that the present study involving 123 patients will be expanded to include more patients, allowing for comparison of the technique across various risk populations. Further large sample studies are also required to investigate how to evaluate the USE images of nodules complicated by diffuse thyroid disease. Finally, owing to the study design, a comparative study on the intra- and inter-examiner variation and reliability was not performed, which may be included in the next phase of the investigation.

The results of the present study indicate that USE demonstrates a high sensitivity for thyroid nodule classification based on elasticity as it assessed the relative stiffness of thyroid nodules accurately. USE may aid the differentiation of benign from malignant thyroid nodules. However, the stiffness of benign and malignant nodules overlaps to a certain extent, particularly in the case of diffuse lesions, fibrosis or calcification surrounding the nodules. In practice, USE may be performed in combination with conventional US to facilitate diagnosis.

\section{Acknowledgements}

The present study was supported by the Shanxi Science and Technology Project Foundation (grant no. 2012k13-02-11).

\section{References}

1. Sipos JA and Mazzaferri EL: Thyroid cancer epidemiology and prognostic variables. Clin Oncol (R Coll Radiol) 22: 395-404, 2010.

2. Davies L and Welch HG: Increasing incidence of thyroid cancer in the United States, 1973-2002. JAMA 295: 2164-2167, 2006.

3. Li N, Du XL, Reitzel LR, Xu L and Sturgis EM: Impact of enhanced detection on the increase in thyroid cancer incidence in the United States: Review of incidence trends by socioeconomic status within the surveillance, epidemiology, and end results registry, 1980-2008. Thyroid 23: 103-110, 2013.

4. Andrioli M and Persani L: Elastographic techniques of thyroid gland: Current status. Endocrine 46: 455-461, 2014.

5. Bhatia KS, Lee YY, Yuen EH and Ahuja AT: Ultrasound elastography in the head and neck. Part II. Accuracy for malignancy. Cancer Imaging 13: 260-276, 2013.

6. Zhan WW: Advances in ultrasound diagnosis of thyroid nodules (J/CD). Chin J Med Ultrasound Electronic Version 8: 1170-1179, 2011.

7. Mansor M, Okasha H, Esmat S, Hashem AM, Attia KA and El-din Hussein H: Role of ultrasound elastography in prediction of malignancy in thyroid nodules. Endocr Res 37: 67-77, 2012.
8. Wong CK and Wheeler MH: Thyroid nodules: Rational management. World J Surg 24: 934-941, 2000.

9. Hegedüs L: Clinical practice. The thyroid nodule. N Engl J Med 351: 1764-1771, 2004.

10. Rago T, Vitti P, Chiovato L, Mazzeo S, De Liperi A, Miccoli P, Viacava P, Bogazzi F, Martino E and Pinchera A: Role of conventional ultrasonography and color flow-doppler sonography in predicting malignancy in 'cold' thyroid nodules. Eur J Endocrinol 138: 41-46, 1998.

11. Tamsel S, Demirpolat G, Erdogan M, Nart D, Karadeniz M, Uluer H and Ozgen AG: Power Doppler US patterns of vascularity and spectral Doppler US parameters in predicting malignancy in thyroid nodules. Clin Radiol 62: 245-251, 2007.

12. Chan BK, Desser TS, McDougall IR, Weigel RJ and Jeffrey RB Jr: Common and uncommon sonographic features of papillary thyroid carcinoma. J Ultrasound Med 22: 1083-1090, 2003.

13. Alexander EK, Marqusee E, Orcutt J, Benson CB, Frates MC Doubilet PM, Cibas ES and Atri A: Thyroid nodule shape and prediction of malignancy. Thyroid 14: 953-958, 2004.

14. Gharib H, Papini E, Valcavi R, Baskin HJ, Crescenzi A, Dottorini ME, Duick DS, Guglielmi R, Hamilton CR Jr, Zeiger MA, et al: American association of clinical endocrinologists and associazione medici endocrinologi medical guidelines for clinical practice for the diagnosis and management of thyroid nodules. Endocr Pract 12: 63-102, 2006.

15. Lyshchik A, Higashi T, Asato R, Tanaka S, Ito J, Mai JJ, Pellot-Barakat C, Insana MF, Brill AB, Saga T, et al: Thyroid gland tumor diagnosis at US elastography. Radiology 237: 202-211, 2005.

16. Ophir J, Céspedes I, Ponnekanti H, Yazdi Y and Li X: Elastography: A quantitative method for imaging the elasticity of biological tissues. Ultrasonic Imaging 13: 111-134, 1991.

17. Havre $\mathrm{R}$ and Gilja $\mathrm{OH}$ : Elastography and strain ratio imaging of the gastrointestinal tract. Eur J Radiol 83: 438-441, 2014.

18. Li P, Song Y and Hu XT: Comparative study on ultrasound elastography and conventional ultrasound for the diagnosis of benign thyroid nodules. Tongji Univ Trans (Med Sci) 31: 88-91, 2010.

19. Cochlin DL, Ganatra RH and Griffiths DF: Elastography in the detection of prostatic cancer. Clin Radiol 57: 1014-1020, 2002.

20. Asteria C, Giovanardi A, Pizzocaro A, Cozzaglio L, Morabito A, Somalvico F and Zoppo A: US-elastography in the differential diagnosis of benign and malignant thyroid nodule. Thyroid 18: 523-531, 2008

21. Rago T, Santini F, Scutari M,Pinchera A and Vitti P: Elastography: New developments in ultrasound for predicting malignancy in thyroid nodules. J Clin Endocrinol Metab 92: 2917-2922, 2007.

22. Lv K, Jiang YX and Zhang JX: Ultrasound diagnosis of thyroid nodules. Chin J Ultrasonography 12: 285-288, 2003.

23. Moon WJ, Jung SL, Lee JH, Na DG, Baek JH, Lee YH, Kim J, Kim HS, Byun JS and Lee DH; Thyroid Study Group, Korean Society of Neuro- and Head and Neck Radiology: Benign and malignant thyroid nodules: US differentiation-multicenter retrospective study. Radiology 247: 762-770, 2008.

24. Youden WJ: Index for rating diagnostic test. Cancer 3: 32-35, 1950.

25. Sande JA, Verjee S, Vinayak S, Amersi F and Ghesani M: Ultrasound shear wave elastography and liver fibrosis: A prospective multicenter study. World J Hepatol 9: 38-47, 2017.

26. Wiest PW, Hartshorne MF, Inskip PD, Crooks LA, Vela BS, Telepak RJ, Williamson MR, Blumhardt R, Bauman JM and Tekkel M: Thyroid palpation versus high-resolution thyroid ultrasonography in the detection of nodules. J Ultrasound Med 17: 487-496, 1998.

27. Dan HJ, Wang Y and Dan HY: Use of real-time ultrasound elastography in the diagnosis of small solitary solid thyroid nodules. China Med Imaging Technol 26: 63-65, 2010.

28. Liu F and Xiao Y: Application of Ultrasonic elastic strain ratio in the diagnosis of benign and malignant thyroid nodules. Chin J Med Ultrasound Electronic Version 7: 671-678, 2010.

29. Jeh SK, Jung SL, Kim BS and Lee YS: Evaluating the degree of conformity of papillary carcinoma and follicular carcinoma to the reported ultrasonographic findings of malignant thyroid tumor. Korean J Radiol 8: 192-197, 2007.

30. Luo BM, Zeng J, Ou B and Zhi H: Effects of the size of the region of interest in breast ultrasound elastography on the diagnostic result. Chin Med Imaging Technol 23: 1330-1332, 2007. 
31. Ophir J, Alam SK, Garra B, Kallel F, Konofagou E, Krouskop T and Varghese T: Elastography: Ultrasonic estimation and imaging of the elastic properties of tissues. Proc Inst Mech Eng H 213: 203-233, 1999.

32. Hong YR, Liu XM, Li ZY, Zhang X, Chen M and Luo Z: Real-time ultrasound elastography in the differential diagnosis of benign and malignant thyroid nodules. J Ultrasound Med 28: 861-867, 2009

33. Bojunga J, Herrmann E, Meyer G, Weber S, Zeuzem S and Friedrich-Rust M: Real-time elastography for the differentiation of benign and malignant thyroid nodules: A meta-analysis. Thyroid 20: 1145-1150, 2010.

34. Vorländer C, Wolff J, Saalabian S, Lienenlüke RH and Wahl RA: Real-time ultrasound elastography-a noninvasive diagnostic procedure for evaluating dominant thyroid nodules. Langenbecks Arch Surg 395: 865-871, 2010.

35. Vidal-Casariego A, López-González L, Jiménez-Pérez A, Ballesteros-Pomar MD, Kyriakos G, Urioste-Fondo A, Álvarez-San Martín R, Cano-Rodríguez I and Jiménez-García de la Marina JM: Accuracy of ultrasound elastography in the diagnosis of thyroid cancer in a low-risk population. Exp Clin Endocrinol Diabetes 120: 635-638, 2012.
36. Magri F, Chytiris S, Capelli V, Alessi S, Nalon E, Rotondi M, Cassibba S, Calliada F and Chiovato L: Shear wave elastography in the diagnosis of thyroid nodules: Feasibility in the case of coexistent chronic autoimmune Hashimoto thyroiditis. Clin Endocrinol (Oxf) 76: 137-141, 2012.

37. Luo S, Kim EH, Dighe M and Kim Y: Thyroid nodule classification using ultrasound elastography via linear discriminant analysis. Ultrasonics 51: 425-431, 2011.

38. Ciledag N, Arda K, Aribas BK, Aktas E and Köse SK: The utility of ultrasound elastography and MicroPure imaging in the differentiation of benign and malignant thyroid nodules. AJR Am J Roentgenol 198: W244-W249, 2012 